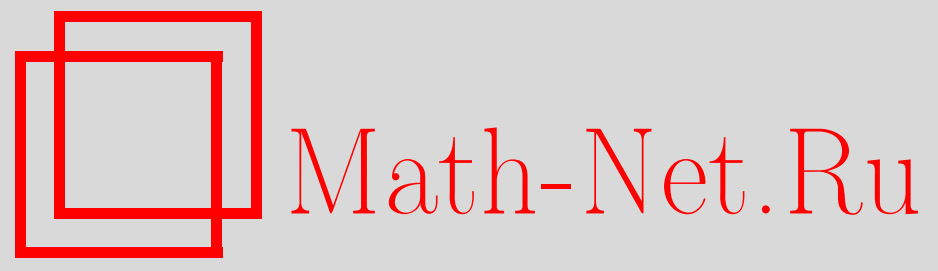

И. А. Чеплюкова, Один случай предельного распределения числа циклических вершин в случайном отображении, Дискрет. матем., 2004, том 16, выпуск 3, 76-84

DOI: https://doi.org/10.4213/dm164

Использование Общероссийского математического портала Math-Net.Ru подразумевает, что вы прочитали и согласны с пользовательским соглашением http://www . mathnet.ru/rus/agreement

Параметры загрузки:

IP: 54.198 .64 .247

26 апреля 2023 г., 13:54:04

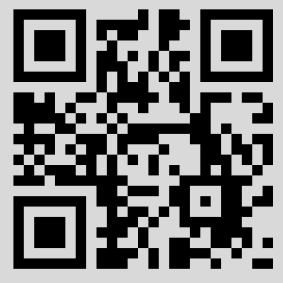




\title{
Один случай предельного распределения числа циклических вершин в случайном отображении
}

\author{
() 2004 г. И. А. Чеплюкова
}

\begin{abstract}
Рассматривается число циклических вершин в случайном однозначном отображении множества объема $n$, граф которого содержит $m$ циклов. Получена теорема, описывающая предельное поведение этой характеристики в случае, когда $n \rightarrow \infty$, $m / \ln n \rightarrow \infty, m / \ln n=O(\ln n)$.

Работа выполнена при поддержке гранта НШ 1758.2003.1 Президента Российской Федерации государственной поддержки ведуших научных школ Российской Федерации.
\end{abstract}

Рассмотрим однозначные отображения множества из $n$ элементов в себя, граф которых содержит $m$ компонент связности. На множестве всех таких отображений введем равномерное распределение вероятностей. В книге [1] получены предельные распределения некоторых характеристик этих случайных отображений. Мы будем придерживаться обозначений [1]. Через $\lambda^{(n, m)}$ обозначим случайную величину, равную числу циклических вершин в случайном отображении с $m$ циклами. Предельное поведение случайной величины $\lambda^{(n, m)}$ в случае ограниченного отношения $m / \ln n$ было исследовано Ю. Л. Павловым в книге [1]. В настоящей работе мы дополним эти результаты, изучив поведение $\lambda^{(n, m)}$ при $n \rightarrow \infty, m / \ln n \rightarrow \infty, m / \ln n=O(\ln n)$.

Введем обозначения

$$
\alpha=\frac{\sqrt{n}}{m}, \quad \beta=\frac{m}{\ln \alpha}, \quad z=\frac{N}{\sqrt{n}}, \quad \mu=\frac{\beta}{2}\left(1-\frac{\ln \ln \alpha}{\ln \alpha}\right) .
$$

Основным результатом данной работы является следующая теорема.

Теорема 1. Если $n \rightarrow \infty, m / \ln n \rightarrow \infty, m / \ln ^{2} n \leqslant C<\infty$, то равномерно относительно чельх $N$, для которых $z-\sqrt{2 \mu}$ лежит в любом фиксированном конечном интервале

$$
\beta^{\mu} e^{-\beta / 2} \sqrt{\pi n} \mathbf{P}\left\{\lambda^{(n, m)} / \sqrt{n}=z\right\}=z^{2 \mu} e^{-z^{2} / 2}(1+o(1))
$$

Замечание 1. Нетрудно проверить, что эта теорема описывает предельное распределение случайной величины $\lambda^{(n, m)}$. Действительно, согласно теореме

$$
S_{n, m, A}=\sum \mathbf{P}\left\{\lambda^{(n, m)}=N\right\}=\frac{\beta^{-\mu} e^{\beta / 2}}{\sqrt{\pi n}} \sum\left(\frac{N}{\sqrt{n}}\right)^{2 \mu} e^{-N^{2} /(2 n)}(1+o(1)),
$$


где суммирование проводится по $N$ таким, что $(\sqrt{2 \mu}-A) \sqrt{n} \leqslant N \leqslant(\sqrt{2 \mu}+A) \sqrt{n}$, а $A$ - некоторая положительная постоянная. Введем переменную $t=N / \sqrt{n}-\sqrt{2 \mu}$. Тогда, используя явный вид $\beta$ и $\mu$, можно показать, что при выполнении условий теоремы

$$
S_{n, m, A}=\frac{\beta^{-\mu} e^{\beta / 2-\mu}(2 \mu)^{\mu}}{\sqrt{\pi n}} \sum_{|t| \leqslant A} e^{-t^{2}}(1+o(1))=\frac{1+o(1)}{\sqrt{\pi n}} \sum_{|t| \leqslant A} e^{-t^{2}} .
$$

Остается заметить, что суммирование по $t$ в последней сумме осуществляется с шагом $1 / \sqrt{n}$, и переходя от суммирования к интегрированию, получаем, что выбором достаточно большого $A$ и $n$ сумму $S_{n, m, A}$ можно сделать сколь угодно близкой к единице.

Ниже мы докажем два вспомогательных утверждения (леммы 1 и 2), с помощью которых будет доказана теорема.

Обозначим через $\lambda^{(n)}$ и $\xi^{(n)}$ соответственно число циклических точек и число циклов случайного отображения множества объема $n$ в себя (без ограничения на число циклов). Нетрудно видеть, что

$$
\mathbf{P}\left\{\lambda^{(n, m)}=N\right\}=\mathbf{P}\left\{\lambda^{(n)}=N \mid \xi^{(n)}=m\right\}=\frac{\mathbf{P}\left\{\lambda^{(n)}=N, \xi^{(n)}=m\right\}}{\mathbf{P}\left\{\xi^{(n)}=m\right\}} .
$$

Для того, чтобы получить предельное распределение случайной величины $\lambda^{(n, m)}$, pacсмотрим отдельно числитель и знаменатель выражения, стоящего в правой части равенства (1).

Обозначим через $\varkappa_{N}$ случайную величину, равную числу циклов в случайной подстановке степени $N$. Нетрудно видеть, что

$$
\mathbf{P}\left\{\xi^{(n)}=m \mid \lambda^{(n)}=N\right\}=\mathbf{P}\left\{\varkappa_{N}=m\right\} .
$$

Поэтому, учитывая, что

$$
\mathbf{P}\left\{\lambda^{(n)}=N, \xi^{(n)}=m\right\}=\mathbf{P}\left\{\lambda^{(n)}=N\right\} \mathbf{P}\left\{\xi^{(n)}=m \mid \lambda^{(n)}=N\right\},
$$

находим, что для числителя правой части соотношения (1) верно равенство

$$
\mathbf{P}\left\{\lambda^{(n)}=N, \xi^{(n)}=m\right\}=\mathbf{P}\left\{\lambda^{(n)}=N\right\} \mathbf{P}\left\{\varkappa_{N}=m\right\} .
$$

В [2] приведено полное описание предельного поведения случайной величины $\varkappa_{N}$. Согласно этим результатам (теорема 4.2.6) при $N \rightarrow \infty, m / \ln N \rightarrow \infty, N / m \rightarrow \infty$

$$
\mathbf{P}\left\{\varkappa_{N}=m\right\}=\frac{(-\ln (1-x))^{m}}{m ! x^{N} \sigma \sqrt{2 \pi m}}(1+o(1)),
$$

где

$$
x=1-\left(\frac{N}{m} \ln \frac{N}{m}\right)^{-1}, \quad \sigma=\frac{N}{m} \sqrt{\ln \frac{N}{m}} .
$$

Для вероятности $\mathbf{P}\left\{\lambda^{(n)}=N, \xi^{(n)}=m\right\}$ справедливо следующее утверждение.

Лемма 1. Если $N \rightarrow \infty, N / m \rightarrow \infty, \bar{\beta}=m / \ln (N / m) \rightarrow \infty, m / \ln ^{2}(N / m) \leqslant C<\infty$, то равномерно относительно $z=N / \sqrt{n} \leqslant n^{1 / 6}$

$$
\frac{\sqrt{2 \pi \bar{\beta}}(m-1) ! n}{e^{\bar{\beta}}(-\ln (1-x))^{m-1}} \mathbf{P}\left\{\lambda^{(n)}=N, \xi^{(n)}=m\right\}=e^{-z^{2} / 2}(1+o(1)),
$$

где х определено в (4). 
Доказательство. Известно (см. [3]), что равномерно относительно $N / \sqrt{n} \leqslant n^{1 / 6}$

$$
\mathbf{P}\left\{\lambda^{(n)}=N\right\}=\frac{N}{n} \exp \left\{-\frac{N^{2}}{2 n}\right\}(1+o(1)) .
$$

Согласно условию леммы $m / \ln (N / m) \rightarrow \infty$, следовательно, при выполнении неравенства $m / \ln ^{2}(N / m) \leqslant C<\infty$ отношение $m / \ln N$ стремится к бесконечности. Поэтому справедливо соотношение (3). Кроме того, нетрудно показать, что

$$
\ln x^{N}=N \ln \left(1-\left(\frac{N}{m} \ln \frac{N}{m}\right)^{-1}\right)=-\frac{m}{\ln (N / m)}+o(1) .
$$

Поэтому утверждение леммы 1 следует из (2)-(6).

Докажем вспомогательную лемму для знаменателя правой части равенства (1).

Лемма 2. Пусть $n \rightarrow \infty, m / \ln (\sqrt{n} / m) \rightarrow \infty, m / \ln ^{2}(\sqrt{n} / m) \leqslant C<\infty$. Тогда

$$
\frac{(m-1) ! \sqrt{2 \beta n}}{\left(-\ln \left(1-x_{1}\right)\right)^{m-1} e^{\beta / 2} \beta^{\mu}} \mathbf{P}\left\{\xi^{(n)}=m\right\}=e^{-\gamma}(1+o(1)),
$$

где

$$
x_{1}=1-\frac{1}{\alpha \ln \alpha}, \quad \alpha=\frac{\sqrt{n}}{m}, \quad \beta=\frac{m}{\ln \alpha}, \quad \gamma=\frac{\beta}{8 \ln \alpha} \ln ^{2}(2 \mu), \quad \mu=\frac{\beta}{2}\left(1-\frac{\ln \ln \alpha}{\ln \alpha}\right) .
$$

Доказательство. Используя (2), находим, что

$$
\mathbf{P}\left\{\xi^{(n)}=m\right\}=\sum_{N=m}^{n} \mathbf{P}\left\{\lambda^{(n)}=N, \xi^{(n)}=m\right\}=\sum_{N=m}^{n} \mathbf{P}\left\{\lambda^{(n)}=N\right\} \mathbf{P}\left\{\varkappa_{N}=m\right\} .
$$

Разобьем область суммирования на девять частей:

$$
\begin{aligned}
& N_{1}=\{N: m \leqslant N<\varepsilon \sqrt{n} / m\}, \\
& N_{2}=\{N: \varepsilon \sqrt{n} / m \leqslant N<\varepsilon \sqrt{n}\}, \\
& N_{3}=\left\{N: \varepsilon \sqrt{n} \leqslant N<\beta^{1 / 2-\delta} \sqrt{n}\right\}, \\
& N_{4}=\left\{N: \beta^{1 / 2-\delta} \sqrt{n} \leqslant N<(\sqrt{2 \mu}-A) \sqrt{n}\right\}, \\
& N_{5}=\{N:(\sqrt{2 \mu}-A) \sqrt{n} \leqslant N \leqslant(\sqrt{2 \mu}+A) \sqrt{n}\}, \\
& N_{6}=\{N:(\sqrt{2 \mu}+A) \sqrt{n}<N<2 \sqrt{2 \mu n}\}, \\
& N_{7}=\left\{N: 2 \sqrt{2 \mu n} \leqslant N<\beta^{1 / 2+\delta} \sqrt{n}\right\}, \\
& N_{8}=\left\{N: \beta^{1 / 2+\delta} \sqrt{n} \leqslant N<n^{2 / 3}\right\}, \\
& N_{9}=\left\{N: n^{2 / 3} \leqslant N \leqslant n\right\},
\end{aligned}
$$

где $\beta$ и $\mu$ определены в (7), а выбор постоянных $\varepsilon, \delta$ и $A$ будет ясен из дальнейшего. Через $S_{i}$ обозначим суммы, соответствующие области суммирования $N_{i}, i=1, \ldots, 9$. Тогда

$$
\mathbf{P}\left\{\xi^{(n)}=m\right\}=\sum_{i=1}^{9} S_{i}
$$


Ниже мы увидим, что основной вклад в сумму, стоящую в правой части равенства (9), дает слагаемое $S_{5}$. Из (3)-(8) находим, что

$$
\begin{aligned}
S_{5}= & \sum_{N_{5}} \mathbf{P}\left\{\lambda^{(n)}=N\right\} \mathbf{P}\left\{x_{N}=m\right\}=\sum_{N_{5}} \frac{N}{n} \exp \left\{-\frac{N^{2}}{2 n}\right\} \frac{(-\ln (1-x))^{m}}{m ! x^{N} \sigma \sqrt{2 \pi m}}(1+o(1)) \\
= & \frac{(1+o(1))\left(-\ln \left(1-x_{1}\right)\right)^{m-1} e^{\beta}}{(m-1) ! n \sqrt{2 \pi \beta}} \sum_{N_{5}}\left(\frac{\ln (N / m)}{\ln (\sqrt{n} / m)}\right)^{m-1 / 2} \exp \left\{-\frac{N^{2}}{2 n}\right\} \\
& \times\left(\left(1+\frac{\ln \ln (N / m)}{\ln (N / m)}\right) /\left(1+\frac{\ln \ln (\sqrt{n} / m)}{\ln (\sqrt{n} / m)}\right)\right)^{m-1} \frac{\exp \{m / \ln (N / m)\}}{\exp \{m / \ln (\sqrt{n} / m)\}}
\end{aligned}
$$

Положим $y=N / \sqrt{n}$. Тогда в условиях леммы, учитывая, что $y<\ln (\sqrt{n} / m)$, нетрудно получить, что

$$
\begin{aligned}
& \ln \left(\left(1+\frac{\ln \ln (N / m)}{\ln (N / m)}\right) /\left(1+\frac{\ln \ln (\sqrt{n} / m)}{\ln (\sqrt{n} / m)}\right)\right)^{m-1} \\
& =m \ln \left(\left(1+\frac{\ln \ln (y \sqrt{n} / m)}{\ln (y \sqrt{n} / m)}\right) /\left(1+\frac{\ln \ln (\sqrt{n} / m)}{\ln (\sqrt{n} / m)}\right)\right)+o(1) \\
& =\frac{m \ln y}{\ln ^{2} \alpha}-\frac{m(\ln \ln \alpha) \ln y}{\ln ^{2} \alpha}+o(1)
\end{aligned}
$$

и

$$
\ln \left(\frac{\ln (N / m)}{\ln (\sqrt{n} / m)}\right)^{m-1 / 2}=\ln \left(1+\frac{\ln y}{\ln \alpha}\right)^{m}(1+o(1))=\frac{m \ln y}{\ln \alpha}-\frac{m \ln ^{2} y}{2 \ln ^{2} \alpha}+o(1) .
$$

Кроме того, полагая $\bar{\beta}=m / \ln (N / m)$, легко показать, что

$$
\frac{e^{\bar{\beta}}}{e^{\beta}}=\exp \left\{-\frac{m \ln y}{\ln ^{2} \alpha}\right\}(1+o(1))
$$

Из (10)-(13) следует, что

$$
\begin{aligned}
S_{5} & =\frac{\left(-\ln \left(1-x_{1}\right)\right)^{m-1} e^{\beta}}{(m-1) ! n \sqrt{2 \pi \beta}} \sum e^{-y^{2} / 2} y^{2 \mu} \exp \left\{-\frac{m \ln ^{2} y}{2 \ln ^{2} \alpha}\right\}(1+o(1)) \\
& =\frac{\left(-\ln \left(1-x_{1}\right)\right)^{m-1} e^{\beta-\gamma}}{(m-1) ! n \sqrt{2 \pi \beta}} \sum y^{2 \mu} e^{-y^{2} / 2}(1+o(1)),
\end{aligned}
$$

где суммирование осуществляется по области $\{y: \sqrt{2 \mu}-A \leqslant y \leqslant \sqrt{2 \mu}+A\}$. Делая замену переменной $t=y-\sqrt{2 \mu}$ и учитывая, что $|t| \leqslant A$ и $\beta / \ln \alpha \leqslant C<\infty$, можно получить, что

$$
S_{5}=\frac{\left(-\ln \left(1-x_{1}\right)\right)^{m-1} e^{\beta / 2-\gamma} \beta^{\mu}}{(m-1) ! n \sqrt{2 \pi \beta}} \sum_{-A \leqslant t \leqslant A} e^{-t^{2}}(1+o(1)) .
$$

Переходя от суммированию к интегрированию и используя тот факт, что суммирование в последней сумме осуществляется с шагом $1 / \sqrt{n}$, находим, что при $n \rightarrow \infty$

$$
S_{5}=\frac{\left(-\ln \left(1-x_{1}\right)\right)^{m-1} e^{\beta / 2-\gamma}}{(m-1) ! \sqrt{2 \pi \beta n}} \beta^{\mu} \int_{-A}^{A} e^{-t^{2}} d y(1+o(1))
$$


При выборе достаточно большого $A$ интеграл, стоящий в правой части равенства (15), можно сделать сколь угодно мало отличающимся от

$$
\int_{-\infty}^{\infty} \exp \left\{-t^{2}\right\} d t=\sqrt{\pi}
$$

Отсюда получаем, что

$$
S_{5}=\frac{\left(-\ln \left(1-x_{1}\right)\right)^{m-1} e^{\beta / 2-\gamma} \beta^{\mu}}{(m-1) ! \sqrt{2 \beta n}}(1+o(1)) .
$$

Оценим сумму $S_{1}$. Известно (см., например, [3]), что $\mathbf{P}\left\{\lambda^{(n)}=N\right\} \leqslant N / n$, а при доказательстве леммы 5.3.12 в [1] показано, что .

$$
\mathbf{P}\left\{\varkappa_{N}=m\right\} \leqslant((m-1) ! N)^{-1}(1+\ln N)^{m-1} .
$$

Отсюда и из (8) следует, что

$$
S_{1} \leqslant \sum_{N<\varepsilon \sqrt{n} / m} \frac{1}{(m-1) ! n}(1+\ln N)^{m-1} \leqslant \frac{\varepsilon}{(m-1) ! \sqrt{n}}\left(1+\ln \frac{\varepsilon \sqrt{n}}{m}\right)^{m-1} .
$$

Возьмем $\varepsilon \leqslant 1 / e$, тогда выполняется неравенство $(1+\ln (\varepsilon \sqrt{n} / m)) \leqslant \ln (\sqrt{n} / m)$ и из (16) и (17) нетрудно получить, что $S_{1}=o\left(S_{5}\right)$.

Рассмотрим слагаемое $S_{2}$. Из (3)-(6) находим, что

$$
\begin{aligned}
S_{2} & \leqslant \frac{\left(\ln ((\varepsilon \sqrt{n} / m) \ln (\varepsilon \sqrt{n} / m))^{m-1}\right.}{n(m-1) ! \sqrt{2 \pi m}} \exp \left\{\frac{m}{\ln (\varepsilon \sqrt{n} / m)}\right\} \sum_{N_{2}}\left(\ln \frac{N}{m}\right)^{1 / 2}\left(1+\frac{\ln \ln (N / m)}{\ln (N / m)}\right) \\
& \leqslant C_{1} \frac{(\ln (\varepsilon \sqrt{n} / m) \ln (\varepsilon \sqrt{n} / m))^{m-1}}{(m-1) ! \sqrt{2 \pi n m}} \exp \left\{\frac{m}{\ln (\varepsilon \sqrt{n} / m)}\right\} \sqrt{\ln \frac{\varepsilon \sqrt{n}}{m}}
\end{aligned}
$$

здесь и далее $C_{1}, C_{2}, \ldots$ обозначают некоторые положительные постоянные. Поскольку при больших $x$ функщия $f(x)=(\ln (x \ln x))^{m-1} \exp \{m / \ln x\}$ возрастает, из (16) и (18) следует, что $S_{2}=o\left(S_{5}\right)$.

Оценим $S_{3}$. Из (3)-(8) легко получить, что

$$
\begin{aligned}
S_{3}=\frac{\left(-\ln \left(1-x_{1}\right)\right)^{m-1} e^{\beta}}{n(m-1) ! \sqrt{2 \pi \beta}} \sum_{N_{3}} \exp \left\{-\frac{N^{2}}{2 n}\right\}\left(\frac{\ln (N / m)}{\ln (\sqrt{n} / m)}\right)^{m-1 / 2} \\
\quad \times\left(\left(1+\frac{\ln \ln (N / m)}{\ln (N / m)}\right) /\left(1+\frac{\ln \ln (\sqrt{n} / m)}{\ln (\sqrt{n} / m)}\right)\right)^{m-1} \frac{e^{\bar{\beta}}}{e^{\beta}}(1+o(1)) .
\end{aligned}
$$

Отсюда и из (11)-(13), учитывая, что $y=N / \sqrt{n}<\beta^{1 / 2-\delta}$, нетрудно найти, что

$$
\begin{aligned}
& S_{3} \leqslant C_{2} \frac{\left(-\ln \left(1-x_{1}\right)\right)^{m-1} e^{\beta}}{(m-1) ! \sqrt{2 \pi \beta n}} \\
& \times \sum_{\varepsilon \leqslant y<\beta^{1 / 2-\delta}} \frac{1}{\sqrt{n}} \exp \left\{-\frac{y^{2}}{2}\right\} \exp \left\{\frac{m \ln y}{\ln (\sqrt{n} / m)}\left(1-\frac{\ln \ln (\sqrt{n} / m)}{\ln (\sqrt{n} / m)}\right)\right\} \\
& \leqslant C_{3} \frac{\left(-\ln \left(1-x_{1}\right)\right)^{m-1} e^{\beta}}{(m-1) ! \sqrt{2 \pi \beta n}} \exp \left\{\frac{m \ln \beta^{1 / 2-\delta}}{\ln (\sqrt{n} / m)}\left(1-\frac{\ln \ln (\sqrt{n} / m)}{\ln (\sqrt{n} / m)}\right)\right\} \sum_{y \geqslant \varepsilon} \frac{1}{\sqrt{n}} e^{-y^{2} / 2},
\end{aligned}
$$


где суммирование по $y$ в последней сумме осуществляется с шагом $1 / \sqrt{n}$. Заменяя суммирование интегрированием, получаем, что

$$
S_{3} \leqslant C_{4} \frac{\left(-\ln \left(1-x_{1}\right)\right)^{m-1} e^{\beta}}{(m-1) ! \sqrt{2 \pi \beta n}} \beta^{\mu} \exp \{-\delta \beta \ln \beta\}
$$

Отсюда и из (16) следует, что $S_{3}=o\left(S_{5}\right)$.

Оценим сумму $S_{4}$. Положим $y=N / \sqrt{n}$. Аналогично тому, как было получено равенство (14), можно показать, что для $S_{4}$ справедливо равенство

$$
S_{4}=\frac{(1+o(1))\left(-\ln \left(1-x_{1}\right)\right)^{m-1}}{(m-1) ! n \sqrt{2 \pi \beta}} e^{\beta-\gamma} \sum_{\beta^{1 / 2-\delta} \leqslant y<\sqrt{2 \mu}-A} e^{-y^{2} / 2} y^{2 \mu} \exp \left\{-\frac{m \ln ^{2} y}{2 \ln ^{2}(\sqrt{n} / m)}+\gamma\right\} .
$$

Пусть $t=y-\sqrt{2 \mu}$, тогда последнее равенство примет вид

$$
\begin{aligned}
S_{4}=\frac{\left(-\ln \left(1-x_{1}\right)\right)^{m-1}}{(m-1) ! n \sqrt{2 \pi \beta}} e^{\beta-\gamma} \sum_{\beta^{1 / 2-\delta}-\sqrt{2 \mu} \leqslant t<-A} \exp \left\{-\frac{1}{2}(t+\sqrt{2 \mu})^{2}\right\} \\
\times(t+\sqrt{2 \mu})^{2 \mu} \exp \left\{-\frac{m \ln ^{2}(t+\sqrt{2 \mu})}{2 \ln ^{2}(\sqrt{n} / m)}+\gamma\right\}(1+o(1)) .
\end{aligned}
$$

Поскольку $|t|<\sqrt{2 \mu}$, нетрудно видеть, что при выполнении условий леммы 2 выполняется неравенство

$$
\exp \left\{-\frac{m \ln ^{2}(t+\sqrt{2 \mu})}{2 \ln ^{2}(\sqrt{n} / m)}+\gamma\right\}<e^{|t|}
$$

Отсюда и из (19) следует, что

$$
S_{4} \leqslant C_{5} \frac{\left(-\ln \left(1-x_{1}\right)\right)^{m-1}}{(m-1) ! n \sqrt{2 \pi \beta}} e^{\beta-\gamma} e^{-\beta / 2} \beta^{\mu} \sum_{t<-A} \exp \left\{-\frac{t^{2}}{2}-t\right\}
$$

Переходя к интегрированию в последнем выражении, получаем, что

$$
S_{4} \leqslant C_{6} \frac{\left(-\ln \left(1-x_{1}\right)\right)^{m-1}}{(m-1) ! \sqrt{2 \pi n \beta}} e^{\frac{\beta}{2}-\gamma} \beta^{\mu} \int_{-\infty}^{-A} \exp \left\{-\frac{t^{2}}{2}-t\right\} d t
$$

Теперь из соотношений (16) и (20) следует, что $S_{4}=o\left(S_{5}\right)$.

Аналогично тому, как было получено равенство (14) для $S_{5}$, нетрудно найти, что для слагаемого $S_{6}$ справедлива оценка

$$
\begin{aligned}
S_{6} & \leqslant C_{7} \frac{\left(-\ln \left(1-x_{1}\right)\right)^{m-1} e^{\beta-\gamma}}{(m-1) ! n \sqrt{2 \pi \beta}} \sum_{\sqrt{2 \mu}+A<y<2 \sqrt{2 \mu}} e^{-y^{2} / 2} y^{2 \mu} \\
& \leqslant C_{8} \frac{\left(-\ln \left(1-x_{1}\right)\right)^{m-1} e^{\beta / 2-\gamma}}{(m-1) ! n \sqrt{2 \pi \beta}} \beta^{\mu} \sum_{A<t<\sqrt{2 \mu}} e^{-t^{2}},
\end{aligned}
$$


где $y=N / \sqrt{n}$, а $t=y-\sqrt{2 \mu}$. Суммирование по $t$ в последней сумме неравенства (21) осуществляется с шагом $1 / \sqrt{n}$. Заменяя суммирование интегрированием, получаем, что

$$
S_{6} \leqslant C_{9} \frac{\left(-\ln \left(1-x_{1}\right)\right)^{m-1} e^{\beta / 2-\gamma}}{(m-1) ! \sqrt{2 \pi n \beta}} \beta^{\mu} \int_{A}^{\infty} e^{-t^{2}} d t .
$$

Отсюда и из (16) следует, что $S_{6}=o\left(S_{5}\right)$.

Рассмотрим сумму $S_{7}$. Используя соотношения (3)-(8) и (11)-(13), нетрудно показать, что для $S_{7}$ выполнено неравенство

$$
S_{7} \leqslant C_{10} \frac{\left(-\ln \left(1-x_{1}\right)\right)^{m-1} e^{\beta-\gamma}}{(m-1) ! n \sqrt{2 \pi \beta}} \sum_{2 \sqrt{2 \mu} \leqslant y \leqslant \beta^{1 / 2+\delta}} e^{-y^{2} / 2} y^{2 \mu} .
$$

Поскольку функция $f(y)=e^{-y^{2} / 2} y^{2 \mu}$ убывает, для $S_{7}$ справедлива оценка

$$
S_{7} \leqslant C_{10} \frac{\left(-\ln \left(1-x_{1}\right)\right)^{m-1} e^{\beta / 2-\gamma}}{(m-1) ! \sqrt{2 \pi n \beta}}(2 \sqrt{2 \mu})^{2 \mu} e^{-4 \mu} \beta^{1 / 2-\delta} .
$$

Отсюда и из (16), учитывая, что

$$
\frac{(8 \mu)^{\mu} \beta^{1 / 2+\delta} \exp \{-4 \mu\}}{(2 \mu)^{\mu} \exp \{-\mu\}} \rightarrow 0,
$$

получаем, что $S_{7}=o\left(S_{5}\right)$.

Оценим слагаемое $S_{8}$. Из (3)-(8) следует, что

$$
\begin{aligned}
& S_{8}=\frac{(1+o(1))\left(-\ln \left(1-x_{1}\right)\right)^{m-1}}{(m-1) ! n \sqrt{2 \pi \beta}} e^{\beta} \sum_{N_{8}}\left(\frac{\ln (N / m)}{\ln (\sqrt{n} / m)}\right)^{m-1 / 2} \\
& \quad \times\left(\left(1+\frac{\ln \ln (N / m)}{\ln (N / m)}\right) /\left(1+\frac{\ln \ln (\sqrt{n} / m)}{\ln (\sqrt{n} / m)}\right)\right)^{m-1} \frac{e^{\bar{\beta}}}{e^{\beta}} e^{-N^{2} /(2 n)}
\end{aligned}
$$

где $\bar{\beta}=m / \ln (N / m)$. Пусть $y=N / \sqrt{n}$. Поскольку для всех $N$ из области $N_{8}$ выполнено неравенство $e^{\bar{\beta}}<e^{\beta}$ и функщия $f(x)=\ln \ln x / \ln x$ при $x \rightarrow \infty$ убывает, нетрудно найти, чTO

$$
S_{8} \leqslant C_{11} \frac{\left(-\ln \left(1-x_{1}\right)\right)^{m-1}}{(m-1) ! n \sqrt{2 \pi \beta}} e^{\beta} \sum_{\beta^{1 / 2+\delta} \leqslant y<n^{1 / 6}}\left(\frac{\ln (y \sqrt{n} / m)}{\ln (\sqrt{n} / m)}\right)^{m} e^{-y^{2} / 2} .
$$

При $y<n^{1 / 6}$ справедливо неравенство $\ln y / \ln \alpha<1$, следовательно,

$$
m \ln \left(1+\frac{\ln y}{\ln \alpha}\right)<\frac{m \ln y}{\ln \alpha} .
$$

Тогда из (22) получаем, что

$$
S_{8} \leqslant C_{12} \frac{\left(-\ln \left(1-x_{1}\right)\right)^{m-1}}{(m-1) ! \sqrt{2 \pi n \beta}} e^{\beta} \sum_{\beta^{1 / 2+\delta} \leqslant y<n^{1 / 6}} \frac{1}{\sqrt{n}} e^{-y^{2} / 2} y^{\beta} .
$$


Рассмотрим сумму

$$
\Sigma=\frac{1}{\sqrt{n}} \sum_{\beta^{1 / 2+\delta} \leqslant y \leqslant n^{1 / 6}} e^{-y^{2} / 2} y^{\beta} .
$$

Положим $t=y-\beta^{1 / 2+\delta}$. Тогда

$$
\Sigma=\frac{1}{\sqrt{n}} \sum_{0 \leqslant t \leqslant n^{1 / 6}-\beta^{1 / 2+\delta}}\left(t+\beta^{1 / 2+\delta}\right)^{\beta} \exp \left\{-\frac{1}{2}\left(t+\beta^{1 / 2+\delta}\right)^{2}\right\},
$$

где суммирование осуществляется с шагом $1 / \sqrt{n}$. Функция

$$
f(t)=\left(t+\beta^{1 / 2+\delta}\right)^{\beta} \exp \left\{-\frac{1}{2}\left(t+\beta^{1 / 2+\delta}\right)^{2}\right\}
$$

на рассматриваемом промежутке убывает, тогда как для $k$-го слагаемого суммы $\Sigma$ справедливо двойное неравенство

$$
\begin{aligned}
\int_{(k-1) / \sqrt{n}}^{k / \sqrt{n}} f(t) d t & <\frac{1}{\sqrt{n}}\left(\frac{k-1}{\sqrt{n}}+\beta^{1 / 2+\delta}\right)^{\beta} \exp \left\{-\frac{1}{2}\left(\frac{k-1}{\sqrt{n}}+\beta^{1 / 2+\delta}\right)^{2}\right\} \\
& <\int_{(k-2) / \sqrt{n}}^{(k-1) / \sqrt{n}} f(t) d t .
\end{aligned}
$$

Просуммировав по $k$ все эти неравенства, получаем, что

$$
\Sigma=\int_{0}^{n^{1 / 6}-\beta^{1 / 2+\delta}} f(t) d t(1+o(1)) .
$$

Из (23)-(25) находим, что

$$
\begin{aligned}
S_{8} & \leqslant C_{13} \frac{\left(-\ln \left(1-x_{1}\right)\right)^{m-1}}{(m-1) ! \sqrt{2 \pi n \beta}} e^{\beta} \int_{\beta^{1 / 2+\delta}}^{\infty} e^{-y^{2} / 2} y^{\beta} d y \\
& \leqslant C_{14} \frac{\left(-\ln \left(1-x_{1}\right)\right)^{m-1}}{(m-1) ! \sqrt{2 \pi n \beta}} e^{\beta} \int_{\beta^{1 / 2+\delta}}^{\infty} \exp \left\{-\frac{y^{2}}{2}\left(\frac{2 \beta \ln y}{y^{2}}+1\right)\right\} d y .
\end{aligned}
$$

Поскольку $y>\beta^{1 / 2+\delta}$, величина $2 \beta \ln y / y^{2}$ стремится к 0 . Поэтому, в силу сходимости последнего интеграла, из (16) и (26) следует, что $S_{8}=o\left(S_{5}\right)$.

Осталось оценить последнюю сумму $S_{9}$. Известно (см., например, [3]), что с ростом $N$ при $N>n^{2 / 3}$ вероятность $\mathbf{P}\left\{\lambda^{(n)}=N\right\}$ уменьшается, поэтому из (5) получаем, что при $N \geqslant n^{2 / 3}$

$$
\mathbf{P}\left\{\lambda^{(n)}=N\right\} \leqslant \mathbf{P}\left\{\lambda^{(n)}=n^{2 / 3}\right\} \leqslant C_{15} n^{-1 / 3} \exp \left\{-n^{1 / 3} / 2\right\} .
$$

Отсюда и из (3)-(6) нетрудно найти, что

$$
\begin{aligned}
S_{9} & \leqslant C_{16} \frac{n^{-1 / 3} \exp \left\{-n^{1 / 3} / 2\right\}\left(-\ln \left(1-x_{1}\right)\right)^{m-1} e^{\beta}}{(m-1) ! \sqrt{\beta}} \sum_{N_{9}}\left(\frac{\ln ((N / m) \ln (N / m))}{\ln (\alpha \ln \alpha)}\right)^{m-1} \frac{1}{N} \\
& \leqslant C_{17} \frac{n^{-1 / 3} \exp \left\{-n^{1 / 3} / 2\right\}\left(-\ln \left(1-x_{1}\right)\right)^{m-1}}{(m-1) ! \sqrt{\beta}} e^{\beta} \sum_{N_{9}}\left(\frac{\ln (N / m)}{\ln (\sqrt{n} / m)}\right)^{m-1} \frac{1}{N} .
\end{aligned}
$$


Поскольку по условию доказываемой леммы $m / \ln ^{2} \alpha \leqslant C<\infty$, для любых $N$ из области $N_{9}$ справедливы неравенства

$$
\frac{\ln (N / m)}{\ln (\sqrt{n} / m)} \leqslant \frac{\ln (n / m)}{\ln (\sqrt{n} / m)}=1+\frac{\ln \sqrt{n}}{\ln (\sqrt{n} / m)} \leqslant 1+\left(1-\frac{\ln \left(C \ln ^{2}(\sqrt{n} / m)\right)}{\ln \sqrt{n}}\right)^{-1}<e .
$$

Из (27) и (28) получаем, что

$$
S_{9} \leqslant C_{18} \frac{\left(-\ln \left(1-x_{1}\right)\right)^{m-1} e^{\beta}}{(m-1) ! \sqrt{\beta n}} \frac{e^{m} \sqrt{n}}{\exp \left\{n^{1 / 3} / 2\right\}} .
$$

Нетрудно проверить, что при выполнении условий леммы 2

$$
\frac{\sqrt{n} e^{m}}{\exp \left\{n^{1 / 3} / 2\right\}} \rightarrow 0
$$

Отсюда и из (16) и (29) следует, что $S_{9}=o\left(S_{5}\right)$.

Из полученных оценок для сумм $S_{1}-S_{9}$ вытекает утверждение леммы 2.

Доказательство теоремы 1. Учитывая, что условия $m / \ln ^{2}(\sqrt{n} / m) \leqslant \tilde{C}<\infty$ и $m / \ln ^{2} n \leqslant C<\infty$ эквивалентны, из равенства (1) и лемм 1 и 2 нетрудно получить, что

$$
\begin{aligned}
\mathbf{P}\left\{\lambda^{(n, m)=}\right. & N\}=\frac{e^{\bar{\beta}}}{e^{\beta}} \sqrt{\frac{\beta}{\bar{\beta}}}\left(\frac{\ln (1-x)}{\ln \left(1-x_{1}\right)}\right)^{m-1} \frac{e^{-z^{2} / 2}}{\sqrt{\pi n}} \beta^{-\mu} e^{\beta / 2+\gamma}(1+o(1)) \\
= & \frac{e^{\bar{\beta}}}{e^{\beta}}\left(\frac{\ln (N / m)}{\ln (\sqrt{n} / m)}\right)^{m-1 / 2}\left(\left(1+\frac{\ln \ln (N / m)}{\ln (N / m)}\right) /\left(1+\frac{\ln \ln (\sqrt{n} / m)}{\ln (\sqrt{n} / m)}\right)\right)^{m-1} \\
& \times \frac{e^{-N^{2} /(2 n)}}{\sqrt{\pi n}} \beta^{-\mu} e^{\beta / 2+\gamma}(1+o(1)) .
\end{aligned}
$$

Отсюда и из (11)-(13) следует утверждение теоремы.

\section{Список литературы}

1. Павлов Ю. Л., Случайные леса. Карельский научный центр РАН, Петрозаводск, 1996.

2. Колчин В. Ф., Случайные графы. Наука, Москва, 2000.

3. Сачков В. Н., Вероятностные методы в комбинаторном анализе. Наука, Москва, 1978.

Статья поступила 30.06.2003. 